\title{
Serum Level of Galectin -3 and Its Relation to Insulin Resistance in Patients with Androgenetic Alopecia
}

N.E.Sorour ${ }^{1}$, A.I.Mustafa ${ }^{1}$, W.A.Abd Elhlim ${ }^{2}$ and A.F.NassrEldin ${ }^{1}$

${ }^{1}$ Dermatology,Venereology and Andrology Dept., Faculty of Medicine, Benha Univ., Benha, Egypt

${ }^{2}$ Lecturer of Clinical \& Chemical Pathology, Faculty of Medicine, Benha Univ., Benha, Egypt

E-Mail:fathiasmaa228@gmail.com

\begin{abstract}
Androgenetic alopecia (AGA) is the most common cause of hair loss in both genders, characterized by follicular miniaturization in a patterned hair loss occurring due to systemic androgen and genetic factors. Galectin-3 is a macrophage-derived instigator of insulin resistance and impaired glucose tolerance, which are two phenotypes associated with obesity-induced type 2 diabetes. Their findings highlight Gal3 as a candidate new therapeutic target to restore insulin sensitivity . to evaluate serum Galectin -3 , fasting insulin and fasting blood glucose levels in patients with androgenetic alopecia and to assess its relation to insulin resistance in androgenetic alopecia patients. This case control study was conducted on 60 patients suffering from Androgentic alopecia (Group A) and 20 apparently healthy individuals of matched age and sex as a control group (Group B). Each patient were subjected to full history taking, complete clinical examination and Estimation of serum levels of Galectin-3 and fasting insulin using enzyme linkedimmunosorbent assay (ELISA) technique, and fasting blood glucose level in both androgenetic alopecia patients and control group. The mean age of AGA patients was 39.9 years. Male patients represented 66.7\%, GAL3 showed excellent AUC (AUC=0.978). At cut off value of 7.9, sensitivity was 93.3\%, specificity was 93.3\%, HOMA IR showed excellent AUC (AUC $=0.967$ ). At cut off value of 1.85 , sensitivity was $93.3 \%$, specificity was $93.3 \%$. Conclusion: higher HOMA IR, GAL3 were considered as independent predictors for AGA development. Higher HOMA IR, GAL3 were considered as independent predictors for more severe AGA in both males a females.
\end{abstract}

Keywords: Galectin -3, Insulin Resistance, Androgenetic Alopecia.

\section{Introduction}

Androgenetic alopecia (AGA) is the most common cause of hair loss in both genders, characterized by follicular miniaturization in a patterned hair loss occurring due to systemic androgen and genetic factors [1].

The age of onset is usually the 3rd and 4th decades, but the hair loss starts immediately after puberty and continues progressively. It affects a variety of psychological and social experiences, and the individual's quality of life [2].

Male androgenetic alopecia occurs as a consequence of the effects of dihydrotestosterone on susceptible hair follicles. Dihydrotestosterone binds to androgen receptors in hair follicles, resulting in gradual transformation of terminal hair follicles to miniaturized hair follicles. The pattern of the hair loss, which typically spares the occipital scalp, reflects regional differences in the sensitivity of scalp follicles to androgens [3].

Female pattern hair ( FPHL) loss is the most common cause of diffuse hair loss in women, It presents with diffuse thinning over the mid-frontal scalp with minimal or no bitemporal recession. Vertex baldness is rare. AGA is associated with several diseases, such as insulin resistance (IR), abnormal serum lipid profiles, and obesity. A positive association between AGA and metabolic syndrome is reported, which is known to increase the risk of type 2 diabetes mellitus and heart diseases [4].

Insulin resistance can contribute to AGA. It plays a pathogenetic role in the miniaturization of hair follicles, in the regulation of androgen metabolism and the hair growth cycle, all of which are relevant to the loss of scalp hair in male-pattern baldness and AGA. Galectin3(Gal3), a member of the multifunctional galectin family, is ubiquitously expressed [5]. It is localized within the cell and secreted into the extracellular space. Extracellular galectin-3 interacts via its C-terminal carbohydrate-recognition domain with the $\beta$-galactoside residues of extracellular matrix and cell surface glycoproteins, whereas interaction of intracellular galectin-3 occurs via peptide-peptide associations mediated by its $\mathrm{N}$-terminus domain [6].

These structural properties enable galectin-3 to exert multiple functions, and galectin- 3 is involved in cell-cell and cell-extracellular matrix adhesion, cell growth and differentiation, apoptosis and angiogenesis. Galectin-3 therefore acts as a broad-spectrum biological response modifier and is involved in tissue fibrosis, tumourigenesis, immunity and the inflammatory response [7].

It can exert several and, at times, opposite functions in a number of pathophysiological processes. Studies have shown that galectin-3 has a profibrotic effect and is involved in the development and progression of heart failure. Galectin-3 is a macrophage-derived instigator of insulin resistance and impaired glucose tolerance, which are two phenotypes associated with obesity-induced type 2 diabetes. Their findings highlight Gal3 as a candidate new therapeutic target to restore insulin sensitivity [8].

The aim of the present study was to evaluate serum Galectin -3, fasting insulin and fasting blood glucose levels in patients with androgenetic alopecia and to assess its relation to insulin resistance in androgenetic alopecia patients. 


\section{Patients and methods}

This case control study was conducted on 60 patients suffering from Androgentic alopecia (Group A) and 20 apparently healthy individuals of matched age and sex as a control group (Group B). Patients were recurrited from the outpatient clinic of Dermatology and Andrology Department of Benha University Hospitals.

The study was approved by the local ethic committee on human research of Benha faculty of medicine. Informed consent was obtained from each individual before being enrolled in the study.

\section{Inclusion criteria}

Diagnosis of androgenetic alopecia will be based on: Clinical findings, pattern of increased hair thinning on frontal/vertex scalp with greater hair density on occipital scalp zone and will be assessed according to the Hamilton-Norwood classification system for men and the Ludwig grade system for women.

\section{Exclusion criteria}

- Patients on hormone replacement therapy (e.g. estrogens, progesterone, testosterone, L-thyroxin, or corticoids) during the last 3 months.

- Patients with previous history of any medications affecting carbohydrate or lipid metabolisms for the prior 3 months.

- Known Patients diabetes mellitus.

- Female patients with history of menstrual irregularities.

- Pregnant and lactating females.

- Patients with baseline disease that causes hair loss. Each patient were subjected to full history taking, complete clinical examination and Estimation of serum levels of Galectin-3 and fasting insulin using enzyme linked-immunosorbent assay (ELISA) technique, and fasting blood glucose level in both androgenetic alopecia patients and control group.

\section{Statistical analysis}

The collected data was revised, coded, tabulated and introduced to a PC using Statistical package for Social Science (IBM Corp. Released 2011. IBM SPSS Statistics for Windows, Version 20.0. Armonk, NY: IBM Corp.). Data were presented and suitable analysis was done according to the type of data obtained for each parameter. Descriptive statistics: Mean, Standard deviation $( \pm$ SD) for numerical data. Frequency and percentage of non-numerical data. Shapiro test was done to test the normality of data distribution. Significant data was considered to be nonparametric. Analytical statistics: Student $\mathrm{T}$ Test was used to assess the statistical significance of the difference between two study group means. For the comparison of the three groups' means, one way analysis of variance (ANOVA) was used. Chi-Square test was used to examine the relationship between two qualitative variables .Fisher's exact test: was used to examine the relationship between two qualitative variables when the expected count is less than 5 in more than $20 \%$ of cells. Correlation analysis: To assess the strength of association between two quantitative variables. The correlation coefficient defines the strength and direction of the linear relationship between two variables. The ROC Curve (receiver operating characteristic) provides a useful way to evaluate the sensitivity and specificity for quantitative diagnostic measures that categorize cases into one of two groups. The optimum cut off point was defined as that which maximized the AUC value.

\section{Results}

The present study included 60 patients with AGA and 20 healthy control groups.

AGA group showed significantly higher HOMA IR, when compared to control group. FBG and insulin did not differ significantly between both groups. Mean GAL-3 in control group was $4.9 \mathrm{ng} / \mathrm{mL}$, while it was $19.4 \mathrm{ng} / \mathrm{mL}$ in AGA cases. AGA group showed significantly higher GAL-3 when compared to control group $(\mathrm{p}<0.001)$ Table $(1)$.

Table (1) Comparison between laboratory data between all studied groups.

\begin{tabular}{|c|c|c|c|c|c|c|c|}
\hline & \multirow{2}{*}{\multicolumn{2}{|c|}{$\begin{array}{c}\text { Control } \mathrm{N}=30 \\
\text { mean }\end{array}$}} & \multicolumn{4}{|c|}{ AGA $\quad \mathbf{N}=\mathbf{3 0}$} & \multirow[t]{2}{*}{$\mathbf{p}$} \\
\hline & & & SD & mean & \pm & SD & \\
\hline FBG (mg/dL) & 90.6 & \pm & 11.6 & 112.5 & \pm & 16.5 & $0.104^{\mathrm{T}}$ \\
\hline Insulin & 5.8 & \pm & 1.9 & 15.1 & \pm & 6.6 & $0.111^{\mathrm{T}}$ \\
\hline HOMA IR & 1.3 & \pm & 0.3 & 4.2 & \pm & 1.3 & $<0.001^{\mathrm{T}}$ \\
\hline Gal-3 & 4.9 & \pm & 1.5 & 19.4 & \pm & 6.3 & $<0.001^{\mathrm{T}}$ \\
\hline
\end{tabular}

SD, standard deviation; T, Student t test.

\section{Discussion}

The current study showed that BMI did not differ significantly between both groups and these results disagreed with the results of the study of Chao-Chun Yang et al., [9], they reported that higher BMI was associated with greater severity of hair loss in men with male-pattern AGA, especially in those with early onset AGA). . This difference can be explained by different control groups of both studies as the present study was conducted on groups of matched age, sex and BMI. 
As regards family history of AGA, the present study showed that there was no statistically significant difference between AGA patients and controls .These results were in agreement with Sreekumar et al., [10]), but in disagreement with Hirsso et al., [11]. These differences could be explained by small sample of patients included in the current study.

the present study showed that there was no significant difference between AGA patients and controls in smoking. These results were in agreement with Arias-Santiago et al., [12], but in disagreement with $\mathrm{Su}$ and Chen, [4], who reported that smoking was found to be significantly associated with AGA. These differences could be explained by small sample included in the current study and female patients where smoking is not common habit in our society.

The present study showed that there was no statistically significant difference between AGA patients and controls in fasting blood glucose levels. These results were in agreement with Mumcuoglu et al., [13], but in disagreement with Arias-Santiago et al., [12], who reported statistically significant difference between AGA patients and controls in fasting blood glucose levels. These results could be explained by higher range of ages (46-60 years old) in their study and exclusion of patients suffer from DM and impaired glucouse tolerance in the current study.

In the present study, serum level of insulin wasn't statistically increased in AGA patients as compared to controls These results were in agreement Nabaie et al. [14], they found no significant difference in fasting insulin levels between patients with AGA and healthy controls, and contributed this finding to the high prevalence of metabolic impairment found in controls as well as in patients with AGA. but in disagreement Arias-Santiago et al., [12], they found that insulin levels were significantly higher in the male and female patients with AGA versus their respective control subjects.

According to mean and standard deviations of HOMA-IR index values, there was statistically significant difference between AGA patients and controls. These results were in agreement with Matilainen et al., [15] who were the first to state an association between insulin resistance and early-onset AGA. Their study was case-control study in men aged 19-50 years showed a strikingly increased risk of hyperinsulinaemia and insulin-resistance-associated disorders such as obesity, hypertension, and dislipidaemia in men with early onset of alopecia $(<35)$, compared with age-matched controls. This finding supports the hypothesis that early androgenetic alopecia could be a clinical marker of insulin resistance.

Insulin is found in hair follicles and may play a role in the regulation of androgen metabolism and the hairgrowth cycle, which are relevant to the loss of scalp hair known as male-pattern baldness. The mechanism by which insulin resistance could contribute to AGA is unclear. It is feasible that either insulin itself or the consequences of insulin resistance have an effect on the circulation of the scalp, causing vasoconstriction and nutrient deficience in the growth area of the hair follicle, increasing the effect of DHT in miniaturization of the follicle. Reduced blood flow might be an important factor in the pathogenesis of early malepattern baldness. It has been shown that compared with a hairbearing scalp, there is microvascular insufficiency in the areas of the bald scalp, which leads to local tissue hypoxia Goldman et al., [16].

IR also leads to the generation of inflammatory mediators and endothelial dysfunction . Insulin increases the release of nitric oxide (NO) from the endothelium at the physiological levels. An increased risk of atherosclerosis in IR cases is thought to be related to the loss of insulin's effect on NO expression Naruse et al., [17].

Results of the present study also were in agreement with Gonzalez-Gonzalez et al.,[18], who found that a relationship exists between insulin resistance and early baldness. The HOMA-IR index was found to be significantly higher among both the patients compared to the controls.

However, results of the current work were in disagreement with Nabaie et al., [14], who found no significant difference in fasting insulin levels between patients with AGA and healthy controls, and contributed this finding to the high prevalence of metabolic impairment found in controls as well as in patients with AGA but in the present study we excluded patients with D.M or other conditions that may predispose to metabolic dysregulation.

To the best of our knowledge, The present study is the first study to evaluate GAL-3 levels in AGA patients aiming at understanding etiopathogenesis of the disease. By measuring serum GAL-3 levels in patients with AGA, which is a disease associated with increased insulin resistance, we evaluated whether GAL-3 level show any correlation with severity of the disease.

\section{Conclusion}

The present study revealed that higher HOMA IR, GAL3 were considered as independent predictors for AGA development. Higher HOMA IR, GAL3 were considered as independent predictors for more severe AGA in both males a females.

\section{References}

[1] J. Paik, J. Yoon, W. Sim, B. Kim, and N. Kim, "The prevalence and types of androgenetic alopecia in Korean men and women," Br. J. Dermatol., Vol.145(1), PP.95-99, 2001.

[2] F. Xu et al., "Prevalence and types of androgenetic alopecia in Shanghai, China: a community-based study," Br. J. Dermatol., Vol.160(3), PP.629-632, 2009.

[3] L. Yip, N. Rufaut, and R. Sinclair, "Role of genetics and sex steroid hormones in male androgenetic alopecia and female pattern hair loss: an update of what we now know," Australas. J. Dermatol., Vol.52(2), PP.81-88, 2011. 
[4] L. Su and T. Chen, "Association of androgenetic alopecia with metabolic syndrome in men: a community-based survey," Br. J. Dermatol., Vol.163(2), PP.371-377, 2010.

[5] J. Nio-Kobayashi, "Tissue-and cell-specific localization of galectins, $\beta$-galactose-binding animal lectins, and their potential functions in health and disease," Anat. Sci. Int., Vol.92(1), PP.25-36, 2017.

[6] K. C. Haudek, K. J. Spronk, P. G. Voss, R. J. Patterson, J. L. Wang, and E. J. Arnoys, "Dynamics of galectin-3 in the nucleus and cytoplasm," Biochim. Biophys. Acta (BBA)General Subj., Vol.1800(2), PP.181-189, 2010.

[7] L. Díaz-Alvarez and E. Ortega, "The many roles of galectin-3, a multifaceted molecule, in innate immune responses against pathogens," Mediators Inflamm., Vol.2(2), PP.34-65, 2017.

[8] H. Ji and X. K. Li, "Oxidative stress in atopic dermatitis. Oxid Med Cell Longev 2016; 2721469," Extern. Resour. Pubmed/Medline Crossref. Vol.3(7), PP.76-85, 2016

[9] C.-C. Yang, F.-N. Hsieh, L.-Y. Lin, C.-K. Hsu, H.M. Sheu, and W. Chen, "Higher body mass index is associated with greater severity of alopecia in men with male-pattern androgenetic alopecia in Taiwan: a cross-sectional study," J. Am. Acad. Dermatol., Vol.70(2), PP.297-302, 2014.

[10] G. P. Sreekumar et al., "Serum androgens and genetic linkage analysis in early onset androgenetic alopecia.," J. Invest. Dermatol., Vol.113(2), PP.277-279, 1999.

[11] P. Hirsso et al., "Association of insulin resistance linked diseases and hair loss in elderly men.
Finnish population-based study.," Cent. Eur. J. Public Health, Vol.14(2), 2006.

[12] S. Arias-Santiago, M. T. Gutiérrez-Salmerón, L. Castellote-Caballero, A. Buendía-Eisman, and R. Naranjo-Sintes, "Androgenetic alopecia and cardiovascular risk factors in men and women: a comparative study," J. Am. Acad. Dermatol., Vol.63(3), PP.420-429, 2010.

[13] C. Mumcuoglu, T. R. Ekmekci, and U. Sema, "The investigation of insulin resistance and metabolic syndrome in male patients with early-onset androgenetic alopecia," Eur. J. Dermatology, Vol.21(1), PP.79-82, 2011.

[14] L. Nabaie et al., "Androgenic alopecia and insulin resistance: are they really related?," Clin. Exp. Dermatology Clin. dermatology, Vol.34(6), PP.694-697, 2009.

[15] V. Matilainen, P. Koskela, and S. KeinänenKiukaanniemi, "Early androgenetic alopecia as a marker of insulin resistance," Lancet, Vol.356(9236), PP.1165-1166, 2000.

[16] B. E. Goldman, D. M. Fisher, and S. L. Ringler, "Transcutaneous PO2 of the scalp in male pattern baldness: a new piece to the puzzle.," Plast. Reconstr. Surg., Vol.97(6), PP.1109-1116, 1996.

[17] K. Naruse et al., "Activation of vascular protein kinase $\mathrm{C}-\beta$ inhibits Akt-dependent endothelial nitric oxide synthase function in obesity-associated insulin resistance," Diabetes, Vol.55(3), PP.691698, 2006.

[18] J. G. González-González et al., "Androgenetic alopecia and insulin resistance in young men," Clin. Endocrinol. (Oxf)., Vol.71(4), PP.494-499, 2009. 